\title{
Termine
}

https://doi.org/10.1515/bd-2020-0085

In der folgenden Terminzusammenstellung werden Fortbildungsseminare und ähnliche Fachveranstaltungen nachgewiesen, die zumindest einer eingeschränkten Öffentlichkeit zugänglich sind, außerdem Jahrestagungen und vergleichbare Anlässe umfassenderer Art. Hingegen können Termine nicht berücksichtigt werden, die ausschließlich lokaler Art oder für einen internen Kreis von Bedeutung sind, zumal in diesem Falle bei den Beteiligten in der Regel die Kenntnis des Termins vorausgesetzt werden kann. Soweit an anderer Stelle im Bibliotheksdienst nähere Informationen über einen bestimmten Termin abgedruckt sind, wird darauf verwiesen. Erstmalig aufgenommene Termine sind mit einem * gekennzeichnet.

\section{Oktober 2020}

6.

7.

14.

\section{November 2020}

11.

18.

25.

\section{Dezember 2020}

2.
Grüner Salon auf der LGS Überlingen. Lesen am See: Verena Roßbacher trifft Franz Michael Felder (s. Heft 5, S. 428)

Digitale virtuelle Bibliothek „Thuebibnet“ für Fortgeschrittene, Erfurt (s. Heft 2, S. 155)

Kreistreffen der Öffentlichen Bibliotheken der Landkreise, Sömmerda (s. Heft 2, S. 158)

Kreistreffen der Öffentlichen Bibliotheken der Landkreise, Hildburghausen (s. Heft 2, S. 158)

Kreistreffen der Öffentlichen Bibliotheken der Landkreise, Ohrdruf (s. Heft 2, S. 158)

Qualitätsmanagement - Fragen und Antworten, Erfurt (s. Heft 2, S. 156)

Fachtagung für Bibliotheksleiter/innen in Öffentlichen Bibliotheken, Erfurt (s. Heft 2, S. 156) 